\title{
Recovery from visual and acoustic hyperaesthesia after mild head injury in relation to patterns of behavioural dysfunction
}

\author{
N Bohnen, A Twijnstra, G Wijnen, J Jolles
}

\begin{abstract}
Patients with head injuries frequently complain of a decreased ability to endure intense light and sound stimuli. The few psychophysical studies that have objectively studied this type of hyperaesthesia have not assessed to what extent patients recover from this hyperaesthesia after mild head injury (MHI). A computerised rating technique was used to assess tolerance to intense sound $(95 \mathrm{~dB})$ and light (1500 lux) stimuli in patients with an uncomplicated MHI. Patients were tested 10 days and five weeks after the injury. Although most patients substantially recovered from both visual and acoustic hyperaesthesia, $25 \%$ of the patients were still not able to endure intense stimuli by five weeks. Analysis of data obtained with two behavioural rating scales (one with post-concussive/cognitive complaints and a second with emotional/vegetative complaints) indicated that visual hyperaesthesia was specifically related to the postconcussive/cognitive complaints scale.
\end{abstract}

There is continuing controversy about the physiogenetic versus psychogenetic origin of the persistent post-concussive syndrome. ${ }^{1}$ As an example of so-called organic evidence, Waddell and Gronwal ${ }^{2}$ demonstrated that MHI patients had a significantly lower threshold tolerance to light and a slightly decreased tolerance to sound one to three weeks after injury compared with healthy controls. Using a computerised method that measures both maximal and submaximal levels of reduced tolerance by applying a graded tolerance scale for each stimulus, we found that MHI patients three to six days after injury had a significantly decreased tolerance to sound stimuli over $71 \mathrm{~dB}$ and to light stimuli over 600 lux.

Unfortunately, no psychophysical studies have measured the recovery from visual and acoustic hyperaesthesia after MHI. In addition, there are no empirical data available about the relationship between hyperaesthesia and different patterns of behavioural symptoms in terms of post-concussive symptoms (PCS), or emotional and functional complaints. It was therefore the aim of this study to investigate: (1) Whether there is significant recovery from visual and acoustic hyperaesthesia following MHI; (2) Whether visual or acoustic hyperaesthesia is related to a partic- ular pattern of post-traumatic behavioural dysfunction.

\section{Materials and methods \\ Subjects}

The criteria for inclusion in the study included a period of unconsciousness ranging from some several seconds to 15 minutes, posttraumatic amnesia for less than 60 minutes, and an EMV score for each patient on admission of 15 . Seventy one patients were selected from a larger population of consecutively admitted patients with mild head injury. Patients $(\mathbf{N}=25$ ) were excluded if one of the following criteria were met: evidence of a focal neurological deficit or of a skull fracture; intoxication at the time of the accident; a concomitant orthopaedic injury; a previous head injury; a history of emotional problems or of hearing or visual problems. Forty six patients (23 females and 23 males; mean age 28.3 (14.9) years) had simple MHI and were selected. The patients were examined within six to 14 days after injury. Three patients did not return for follow up assessment five weeks after injury (four to six weeks) and were also excluded. The 43 patients were individually matched for age and sex with non-concussed control subjects who were recruited from a pool of healthy volunteers (mean age 29.2 $(14 \cdot 2)$ years). The study was approved by the medical ethical council of the University Hospital and all subjects gave their informed consent.

\section{Post-concussive symptoms}

A checklist of PCS was completed, which included items such as headache, dizziness, nausea, difficulties with concentration and memory, fatigue, sleep disturbances and blurred vision. The symptoms were scored for the absolutely or relatively increased appearance after the injury in comparison with before the injury.

Five weeks after injury the patients were divided into a group with persistent PCS (at least one PCS) and a group without PCS.

Procedure of rating the magnitude of tolerance Light and sound stimuli of different intensities were presented using an IBM-XT personal computer as previously described. ${ }^{3}$ Briefly, the computer contained a parallel interface and controlled both a tone generator (calibrated for $100 \mathrm{~Hz}$ ) with varying amplitude and a $50 \mathrm{~W}$ 
tungsten-halogen lamp. The tone generator was connected to a pair of ATH-910 earphones (Audiotechnical) with noise reducing caps.

Five intensities of sound and light were presented $(57,71,81,89$ and $95 \mathrm{~dB}$ for sound and 440,500,600, 1000 and 1500 lux for light). Each of the five intensities was randomly repeated eight times. Each stimulus was presented for four seconds and was followed by a constant interval. During this interval, the subject was asked to evalute the preceding stimulus on a seven-point key board, ranging from totally tolerable/bearable (score: $s 0$ ), via very mildly (s1), mildly (s2), moderately (s3), moderately to severely (s4), severely (s5), to totally unbearable (s6). After the session, the median tolerance value was calculated for each intensity level. Each subject was seated to look into the centre of the light source, which was one metre from their eyes. The $95 \mathrm{~dB}$ and 1500 llux intensities were chosen as the principal parameters in this study.

\section{Patterns of behavioural dysfunction}

The 71 consecutively admitted MHI patients filled in a questionnaire within 10 days after the trauma. There were 26 questions covering post-concussive, cognitive, emotional and psychovegetative complaints. This information was used to draw up different rating scales. A principal component analysis (with varimix rotation $)^{4}$ on the total item pool revaled that there were two relevant factors. Factor I (postconcussive/cognitive complaints) consisted of 11 items (with a factor loading of plus or minus 0.45 or higher) evaluating cognitive-energetic and habitual PCS, such as headache, dizziness, fatigue, intolerance to light or sound, and problems with decreased work performance. Factor II (emotional/vegetative complaints) included 14 items of rather aspecific psychovegetative symptoms, such as complaining of indigestion, dyspnoea, sweating and feeling faint, as well as of items of depression and emotional lability.

As the aim of the study was to correlate different patterns of behavioural symptoms with objectively assessed tolerance to light and sound, the two items of factor I referring to subjectively experienced intolerance to light and sound were removed to avoid bias of partial autocorrelation. The internal consistency of the two groups of items was calculated according to the classical test theory of item analysis (Cronbach's alpha). Cronbach's alpha for the first group (9 items) was 0.88 and 0.86 for the second group. These two groups of items thus both form a scale. ${ }^{5}$ Total scores were calculated by summing the original scores for the selected items per factor. The response to each question was scored according to the intensity of the endorsed complaints (4 levels).

\section{Results}

Ten days after the injury, MHI patients $(\mathrm{N}=$ 43) had a significantly lower tolerance for both $95 \mathrm{~dB}$ sound (Wilcoxon's $\mathrm{z}=3 \cdot 1, \mathrm{p}<0.01$ ) and 1500 lux light $(z=4.30 ; p<0.001)$ than the non-concussed controls. There was significant recovery from the reduced tolerance to light (Friedman Chi square $=5 \cdot 75, \mathrm{df} 1, \mathrm{p}<$ $0 \cdot 05$ ) and sound (Chi square $=9 \cdot 25, \mathrm{df1}, \mathrm{p}<$ $0.01)$. Nevertheless, the proportion of patients with considerable to completely reduced tolerance (scores 5-6) to $95 \mathrm{~dB}$ were $17 / 43$ at 10 days and $11 / 43$ at five weeks (compared with $1 / 43$ in the non-concussed controls). For 1500 lux light, these proportions were 18/43 and $10 / 43$ at the same time points, compared with $2 / 43$ in the controls.

Only three patients were totally free of postconcussive symptoms 10 days after injury whereas this number increased to 12 after five weeks. As can be seen from the table, it was found that patients with PCS at five weeks ( $n=$ 31) had a significantly decreased tolerance to light (Wilcoxon's $z=-1.78, p<0.05$ ) and sound $(z=-2.76, p<0.01)$ compared with the patients without PCS.

The scores on the two behavioural rating scales were compared with the magnitude of the visual and acoustic hyperaesthesia in the total group of patients with uncomplicated MHI ( $n=43)$. Correlational data (Spearman's rank correlation: Rs) indicated that reduced tolerance to sound 10 days after the trauma was significantly correlated with higher scores on both the post-concussive/cognitive scale (Rs $=0.52, \mathrm{p}<0.001)$ and the emotional vegetative scale $(R s=0.43, p<0.01)$. Reduced tolerance to light was only significantly correlated with higher scores on the post-concussive/cognitive scale (Rs $=0.44 ; p$ $<0.01$ ), and not with the scores on the emotional/vegetative scale ( $\mathrm{Rs}=0 \cdot 18, \mathrm{~ns})$.

Lastly, we investigated whether there was a relationship between the extent of recovery from visual and acoustic hyperaesthesia (scores at five weeks relative to those at 10 days) and the scores obtained with the two behavioural rating scales at the two time points. Therefore, difference scores (five weeks minus 10 days) were calculated for each parameter and used for correlational analysis. It was found that there was no significant correlation between the differences in tolerance to sound with the difference scores for either of the two scales (Rs $=0 \cdot 24$, ns for the post-concussive/cognitive scale; Rs $=0.08$ for the emotional/vegetative scale). In contrast, differences in tolerance to light were significantly correlated with the differences in scores measured with the postconcussive/cognitive scale $(R s=0.36, p<$ 0.05 ) but not with the changes measured with the emotional/vegetative scale ( $R s=0 \cdot 24, n s)$.

Table Mean (SD) levels of tolerance to $95 \mathrm{~dB}$ sound Table Mean (SD) levels of tolerance to $95 \mathrm{~dB}$ sound without persistent post-concussion symptoms (PCS) five weeks after injury

\begin{tabular}{llll}
\hline & $N$ & $95 d B$ & 1500 lux \\
\hline Patients with PCS & 31 & $3.63(1.63)^{\star \star}$ & $3.38(1.62)^{\star}$ \\
Patients without PCS & 12 & $2.05(1.39)$ & $2.36(1.86)$ \\
\hline
\end{tabular}

${ }_{\star \star}^{*} \mathrm{p}<0.05$ 


\section{Discussion}

Assessment of visual and acoustic hyperaesthesia can be used as an objective measure of MHI. ${ }^{23}$ Although we found that most patients recovered from their decreased tolerance to intense light and sound stimuli, $25 \%$ of the patients with MHI still demonstrated a considerable to completely reduced tolerance to $95 \mathrm{~dB}$ sound or 1500 lux light at five weeks after MHI. In addition, patients who still complained of persisting PCS tolerated the intense light and sound stimuli less well than those patients who had no PCS. This agrees with the findings of Jonsson et $\mathrm{al}^{6}$ who found that MHI patients with persistent post-concussional symptoms three months after injury had a significantly lower ability to endure intense sound and light stimuli than controls. Our results indicate that assessment of visual and acoustic hyperaesthesia can be used as an objective parameter for the evaluation of the recovery of MHI patients.

Finally, it appeared that the more organic pattern of post-concussive/cognitive symptoms was particularly associated with the extent of the visual hyperaesthesia but not with the pattern of emotional/vegetative complaints. Whether assessment of visual hyperaesthesia reflects recovery from post-traumatic brain dysfunction better than assessment of acoustic hyperaesthesia cannot be concluded from this study.

1 Lishman WA. Physiogenesis and psychogenesis in the 'postconcussional syndrome'. $B r F$ Psychiatry 1988;153: 460-9.

2 Waddell PA, Gronwall DMA. Sensitivity to light and sound following minor head injury. Acta Neurol Scand 1984; 69:270-6.

3 Bohnen N, Twijnstra A, Kroeze J, Jolles J. A psychophysical method for assessing visual and acoustic hyperaesthesia in method for assessing visual and acoustic hyperaest

4 SAS User's Guide: Statistics Vol 5. Cary, NC: Sas Institute I 1985.

5 Novick MR. The axioms and principal results of classical test theory. F Mathematical Psychol 1966;3:1-18.

6 Jonsson C-O, Lidvall $H$, Mälhammer G. An exploratory psychological study of the post-traumatic cerebral syndrome. Acta Neurol Scand 1967;43:158-66. 